\title{
Foreign Gas Broadening of the Lines of Hydrogen Chloride and Carbon Monoxide
}

\author{
Earle K. Plyler and Robert J. Thibault
}

(June 7, 1962)

\begin{abstract}
The Lorentz line shape formula is applied in the determination of half-width parameters due to foreign gas broadening. Various pressures of $\mathrm{SO}_{2}$ and $\mathrm{CO}_{2}$ are added to $\mathrm{HCl}$ and $\mathrm{CO}$, respectively. The (1-0) and $(2-0) \mathrm{HCl}$ bands, as well as the (2-0) $\mathrm{CO}$ band, are investigated, and there is found to be a very definite relationship between the half-width and the rotational quantum number, $J$. No error of more than 5 percent should be expected for the values of $\gamma^{0}$ presented. Some pressure-shifts are observed for $\mathrm{HCl}$, but no quantitative determinations are made.
\end{abstract}

\section{Introduction}

In recent years an interest has developed in the area of pressure-broadening effects. Several studies have been made in the field $[1,2,3],{ }^{1}$ a oreat number of which have been restricted to the self-broadening of gases. There have also been some which have dealt with the broadening effects induced by the addition of a foreign gas to the sample under investigation. Many of these experiments, including the present one, have been performed using highly polar hydrogen chloride (see refs. 1, 2), because of its relative simplicity and freedom from overlapping. The proximity of the isotopic doublet $\mathrm{HCl}^{35}$ and $\mathrm{HCl}^{37}$ also allows one to establish a calibration reference without resorting to extrinsic methods.

Several similar studies have also been made with regard to carbon monoxide (see ref. 3). These have, for the most part, been limited to self-broadening.

The results of recent work on both $\mathrm{HCl}$ and $\mathrm{CO}$ have shown in general that the broadening effect becomes greater as one goes from a higher to a lower value of the rotational quantum number, $J$. This holds true for both self-broadening and foreign gas broadening.

The parameters of interest in such an investigation as this are the line strengths, shapes, and widths. From a knowledge of the line strength, $S$, information can be obtained regarding the quantum-mechanical transition probability. The width and shape of a line are directly related to the specific intermolecular forces involved in the broadening process. The relationship between these parameters may most easily be seen by referring to the well-known Lorentz line shape formula

$$
k=\frac{S}{\pi} \frac{\gamma}{\left(\nu-\nu_{0}\right)^{2}+\gamma^{2}}
$$

where $k$ is the extinction coefficient and $\gamma$ is the Lorentz half-width.

The strengths of the individual lines of both $\mathrm{HCl}$ and $\mathrm{CO}$ have been measured by several investigators

\footnotetext{
1 Figures in brackets indicate the literature references at the end of this paper.
}

(see refs. 1, 2, 3). Emphasis in the present work, therefore, is placed on the evaluation of the halfwidth parameter, $\gamma$, of lines broadened by the addition of a foreign gas to the sample in question. A nonlinear molecular gas, namely sulfur dioxide, is added to $\mathrm{HCl}$, while carbon dioxide is the foreign gas used in the $\mathrm{CO}$ broadening study. Both the $(1-0)$ and $(2-0)$ bands of $\mathrm{HCl}$, as well as the $(2-0)$ band of $\mathrm{CO}$, are extensively investigated. Some measurements of the (1-0) $\mathrm{CO}$ band are made to be used as a comparison with the values obtained from the harmonic band.

A major difficulty encountered in work of this kind is the deviation of a spectral line from its true shape caused by instrumental distortion. Although the resolving power of the instrument used in this work is quite high, it is still necessary to modify the observed half-width values by the inclusion of a slit width correction. In certain cases, notably in the $(2-0)$ band of $\mathrm{HCl}$, these corrections become as large as 20 percent of the line half-width.

No attempt is made here to determine the absolute positions of the individual spectral lines, since this has been done quite accurately in the past $[4,5,6]$.

\section{Experiment}

The spectrometer used in the present experiment is the high resolution grating spectrometer previously described by Plyler and Blaine [7]. The instrument was provided with double pass optics and had a total path length of approximately $13 \mathrm{~m}$.

For the $\mathrm{HCl}$ study the source of radiation employed for both the $(1-0)$ and the $(2-0)$ bands was a $300 \mathrm{w}$ Sylvania enclosed zirconium are, while a Nernst glower was used for the investigation of $\mathrm{CO}$ broadening in the fundamental band. A 10,000 lines/in. Bausch and Lomb grating was employed in the first order for the $(1-0) \mathrm{HCl}$ band and for the $(2-0) \mathrm{CO}$ band. The second order of the grating was used for the $(2-0)$ band of HCl. Suitable optical transmission filters were inserted in the path of radiation to insure the purity of the desired grating order. 
The detector used in all cases was a liquid-nitrogen cooled lead sulfide photodetecting cell. A satisfactory signal-to-noise ratio of about 50 was obtained with a spectral slit width of $0.04 \mathrm{~cm}^{-1}$ in the $\mathrm{HCl}$ fundamental, $0.075 \mathrm{~cm}^{-1}$ in the $\mathrm{HCl}$ harmonic, and 0.05 in the $(2-0) \mathrm{CO}$ band.

Two 10-cm absorption cells, one monel and the other glass, were employed. Both had sapphire windows of equal transmission. Both cells were measured empty before and after use, in order to corroborate the assumption that the background contribution was negligible.

Measured pressures of $\mathrm{HCl}$ and $\mathrm{SO}_{2}$ were added to the cells. The pressures covered a range of from 0.2 to $20 \mathrm{~cm}(\mathrm{Hg})$ of $\mathrm{HCl}$, and from 35 to $132 \mathrm{~cm}$ $(\mathrm{Hg})$ of $\mathrm{SO}_{2}$. These pressure combinations were then measured through both isotopes of each $\mathrm{HCl}$ line at room temperature. While scanning a line the grating was rotated by approximately $18 \mathrm{sec}$ of angle per minute of time, which provided an accurate reproduction of spectral energy distribution.

Utilizing the scanning method described above, a suitable spectrum of $\mathrm{CO}+\mathrm{CO}_{2}$ was then obtained for various pressure combinations, ranging from 19 to $45 \mathrm{~cm}(\mathrm{Hg})$ of $\mathrm{CO}$, and from 42 to $158 \mathrm{~cm}(\mathrm{Hg})$ of $\mathrm{CO}_{2}$.

A mounting method was devised whereby an $\mathrm{SO}_{2}$-broadened $\mathrm{HCl}$ line could be compared with a self-broadened line at the same $\mathrm{HCl}$ pressure. This system consisted of merely shifting the cells alternately into and out of the radiation path while the spectrometer was in continuous operation. This method of rapid alternation was used to investigate the pressure shift between the two lines. In this case the result was, effectively, the superposition of one line upon the other.

\section{Results}

Figure 1 shows a typical self-broadened and $\mathrm{SO}_{2}$ broadened $\mathrm{HCl}$ isotopic doublet. The peak extinction was measured on each $\mathrm{HCl}^{35}$ line wherever feasible. Where the observed absorption approached 100 percent, the $\mathrm{HCl}^{37}$ line was measured, and the result multiplied by the chlorine isotopic abundance ratio, $\left(\frac{\mathrm{Cl}^{35}}{\mathrm{Cl}^{37}} \approx 3.07\right)$, to determine the extinction coefficient of the $\mathrm{HCl}^{35}$ line in question. This abundance ratio was checked using doublets where both isotopes were well below 100 percent absorption, and seemed to be a good approximation.

The extinction at several values of $\left|\nu-\nu_{0}\right|$ was then calculated from the Lorentz line shape formula eq (1). This equation has been shown by Benedict, Herman, Moore, and Silverman (see ref 1) to be valid far enough from the resonant frequency to render it serviceable in the present work. It was then possible to measure directly on the trace the half-width parameter, $\gamma$. This was done at many points on each line corresponding to different extinction percentages.

The average deviation of the several values of $\gamma$ determined for each line was found to be no more than 2 percent. It should be noted that measurements in all cases were made on both the $\mathrm{P}$ and the $\mathrm{R}$ branches. The results were found to be well within the same limits for both branches.

As has previously been mentioned, a correction for the slit width was determined using the corrections given by Izatt [8]. These varied from 0.5 percent to 20 percent, depending on the pressure and on the spectral slit width.

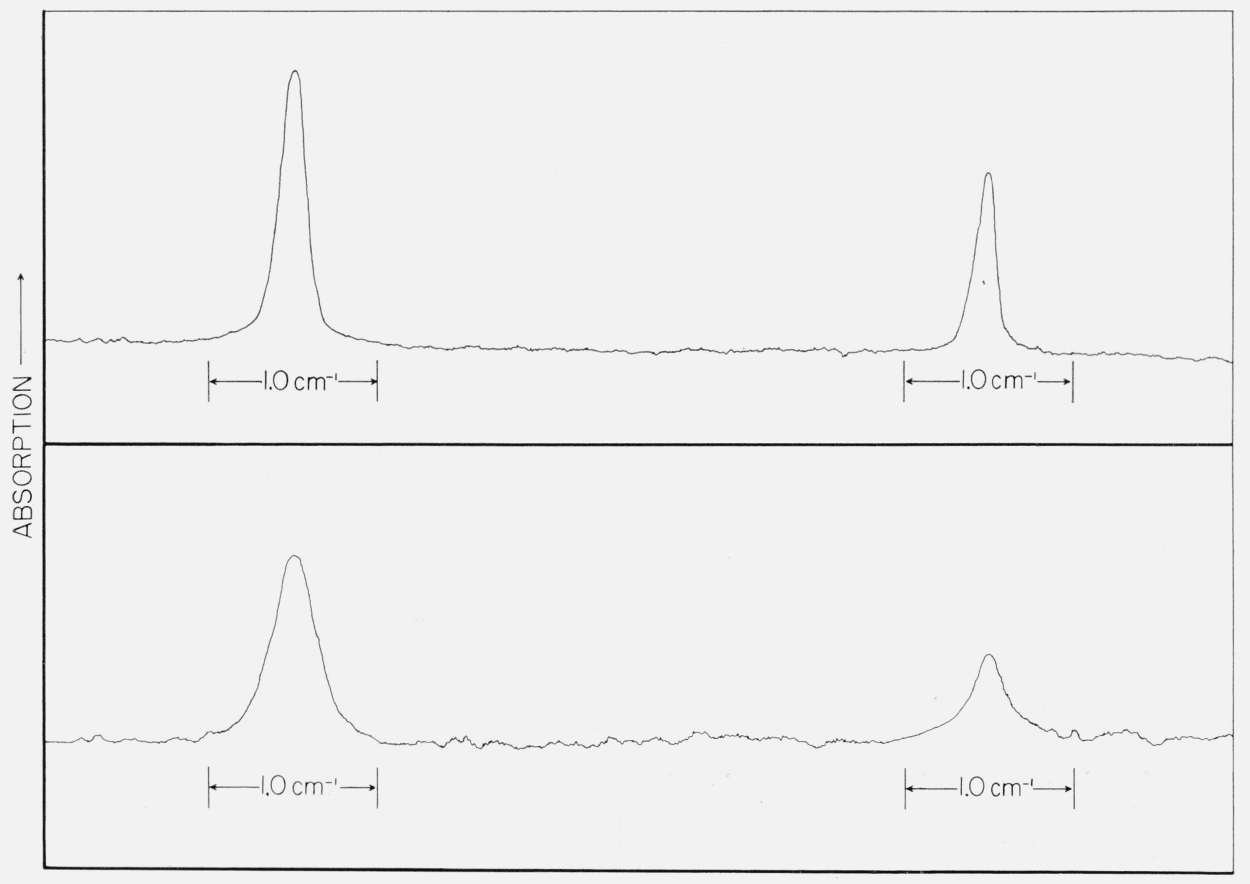

Figure 1. The upper curve shows the $\mathrm{HCl}$ line $R_{2}$ of the 2-0 band with a pressure of $11 \mathrm{~cm}(\mathrm{Hg})$. The lower curve shows the same line of $\mathrm{HCl}$ at $11 \mathrm{~cm}(\mathrm{Hg})$ pressure with $35 \mathrm{~cm}(\mathrm{Hg})$ of $\mathrm{SO}_{2}$ added. 
The values of the half-width parameter given by Benedict et al. (see ref 1) for self-broadened $\mathrm{HCl}$ were then employed to determine the effect of $\mathrm{SO}_{2}$ broadening on the $\mathrm{HCl}$ line in question. The equation used was the following:

$$
\gamma_{\left(\mathrm{SO}_{2}\right)}^{0} P_{\left(\mathrm{SO}_{2}\right)}=\gamma_{(\mathrm{tot})}^{0} P_{(\mathrm{tot})}-\gamma_{(\mathrm{HCl})}^{0} P_{(\mathrm{HCl})} .
$$

The contribution of the self-broadening term can be seen in table 1 . Table 2 gives the average values of $\gamma_{\left(\mathrm{SO}_{2}\right)}^{0}$, where

$$
\gamma_{\left(\mathrm{SO}_{2}\right)}^{0}=\frac{\gamma_{\left(\mathrm{SO}_{2}\right)}}{P_{\left(\mathrm{SO}_{2}\right)}}
$$

The numbers in parentheses in table 2 denote the total number of measurements averaged in each case. A graph of $\gamma_{\left(\mathrm{SO}_{2}\right)}^{0}$ versus $|m|$ is given in figure 2 for both the (1-0) and the (2-0) bands of $\mathrm{HCl}$.

Again using the Lorentz shape formula, the line strengths of several $(1-0) \mathrm{HCl}$ doublets were calculated. When corrected for the slit width, these were in general agreement with those published by Babrov et al. [9].

TABLE 1. Observed half-width, $\gamma$, and half-widths corrected for self-broadening for several values of $|m|$

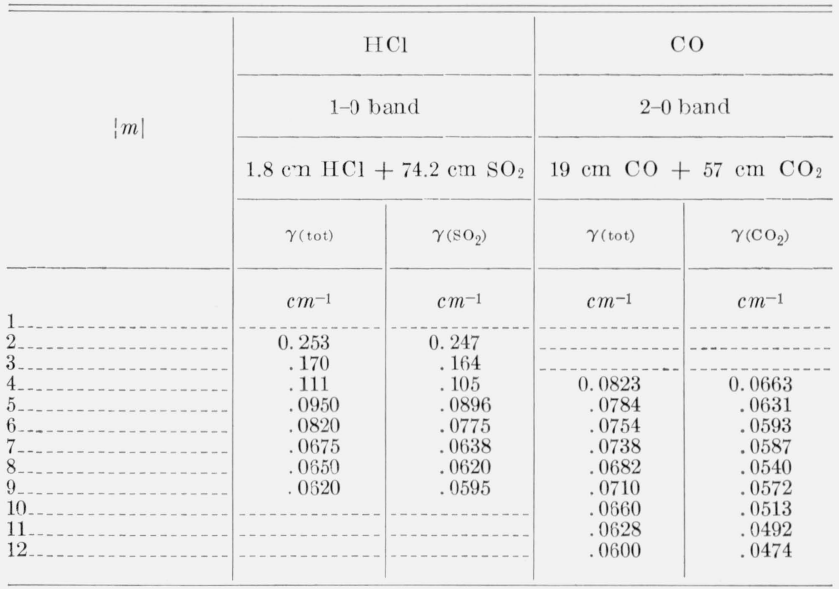

TABLE 2. Half-widths $\gamma_{\left(\mathrm{SO}_{2}\right)}^{\circ}$ for $\mathrm{HCl}$, and $\gamma_{\left(\mathrm{CO}_{2}\right)}^{\circ}$ for $\mathrm{CO}$

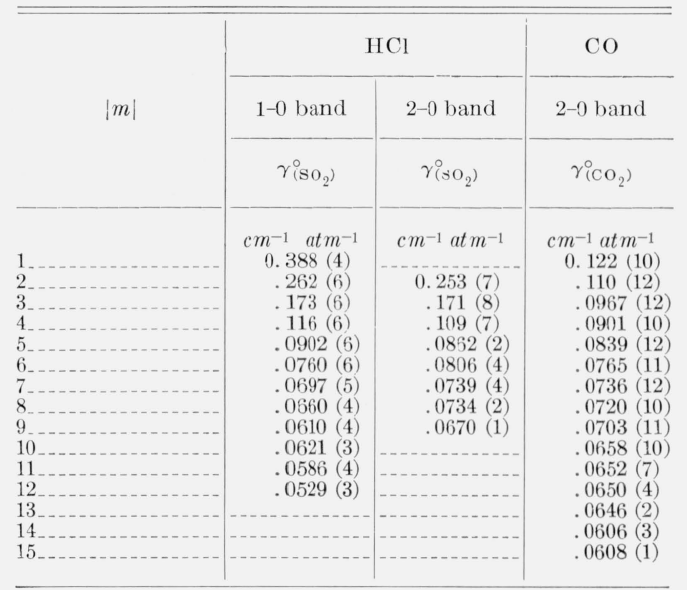

The method described above for $\mathrm{HCl}$ was also used in the determination of $\gamma_{\left(\mathrm{CO}_{2}\right)}$ for several $|m|$ values of the (2-0) $\mathrm{CO}$ band. In this calculation the $\gamma_{\text {(ico) }}$ values employed were those published by Benedict et al. (see ref 3 ).

The Lorentz shape formula was seen to be valid for $\left|\nu-\nu_{0}\right|<4 \mathrm{~cm}^{-1}$, and, as was the case for $\mathrm{HCl}$, no wing correction was included in the values of $\gamma_{\left(\mathrm{CO}_{2}\right)}$ listed in table 2. These $\gamma_{(\mathrm{CO})}^{\circ}$ values were obtained through a method similar to that employed in the case of $\mathrm{HCl}$. The following equation applies:

$$
\gamma_{\left(\mathrm{CO}_{2}\right)}^{\circ} P_{\left(\mathrm{CO}_{2}\right)}=\gamma_{(\mathrm{tot})}^{\circ} P_{(\mathrm{tot})}-\gamma_{(\mathrm{CO})}^{\circ} P_{(\mathrm{CO})}
$$

where the average deviation of the $\gamma_{(t o t)}$ was found to be of the same order as that reported for $\gamma_{(t o t)}$ in the $\mathrm{HCl}$ study $(\leq 2 \%)$.

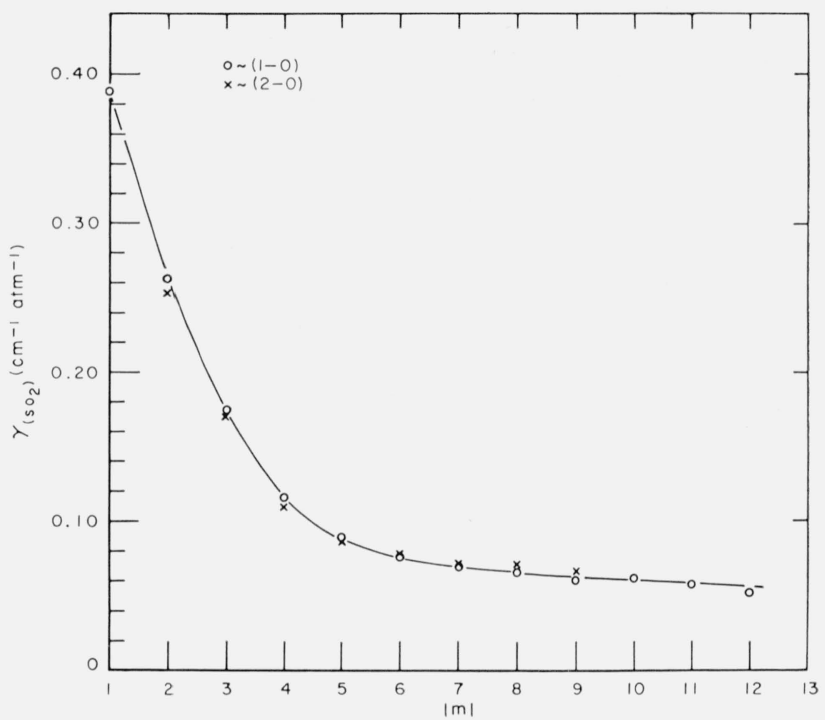

Figure 2. The half-widths, $\gamma^{\circ}\left(\mathrm{sO}_{2}\right)$, of $\mathrm{HCl}$ broadened by $\mathrm{SO}_{2}$ plotted as a function of the rotational level, $|\mathrm{m}|$.

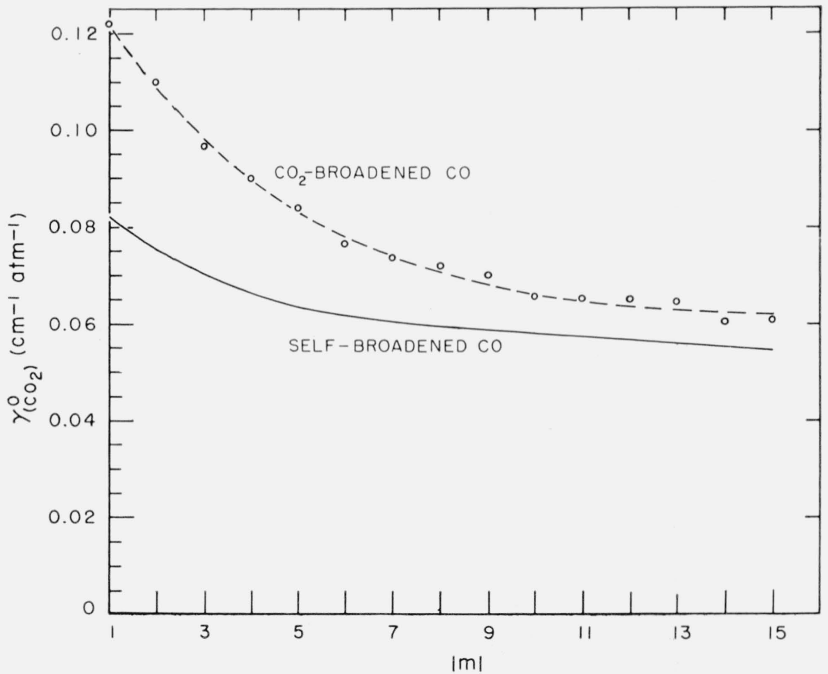

Figure 3. The half-widths, $\gamma^{\circ}{ }_{\left(\mathrm{CO}_{2}\right)}$, of $\mathrm{CO}$ broadened by $\mathrm{CO}_{2}$ plotted as a function of the rotational level, $|\mathrm{m}|$.

Also, self-broadened $\mathrm{CO}$ curve, taken from Benedict et al. (see ref 3). 
The effect of self-broadening introduced by eq (4) is somewhat larger for $\mathrm{CO}$ than it was in the case of $\mathrm{HCl}$, as is evident from table 1.

The average values of $\gamma_{\left(\mathrm{CO}_{2}\right)}^{\circ}$ are shown in figure 3 , along with the $\gamma_{(\mathrm{CO})}^{\circ}$ self-broadening parameters published by Benedict et al. (see ref 3 ), for ready comparison.

As has been mentioned, some measurements of $\mathrm{CO}_{2}$-broadened $\mathrm{CO}$ fundamental lines were made. These agreed within \pm 5 percent with the $\gamma^{\circ}{ }_{(\mathrm{CO} 2)}$ values obtained for the $\mathrm{CO}(2-0)$ band. This is well within experimental error, since the presence of unidentified overlapping bands made the fundamental quite difficult to measure.

Throughout this work it has been assumed that the broadening effect is linear with pressure. This was found to be the case, as is illustrated in figure 4 .

\section{Conclusion}

Figures 2 and 3 show a regular correspondence between the half-width broadening parameter and the rotational quantum number, $J$, as was to be expected. This very definite relationship has been explained by several investigators $[10,11]$. There was no appreciable difference demonstrated between the broadening effects on the fundamental and the harmonic bands in either of the two molecules under investigation.

Since insufficient data were obtained regarding the pressure shift effect induced on $\mathrm{SO}_{2}$-broadened $\mathrm{HCl}$ lines, no conclusions regarding this aspect of the present work will be drawn at this time. It may be noted, however, that a red shift was observed $\left(\sim 0.01 \mathrm{~cm}^{-1} / \mathrm{atm}\right)$ in some of the lines of the $(2-0)$ $\mathrm{HCl}$ band, as has been reported by Hirshfeld, Jaffe, and Kimel [12].

The authors express their gratitude to W. S. Benedict for his many helpful comments and suggestions regarding this paper.

(Paper 66A5-177)

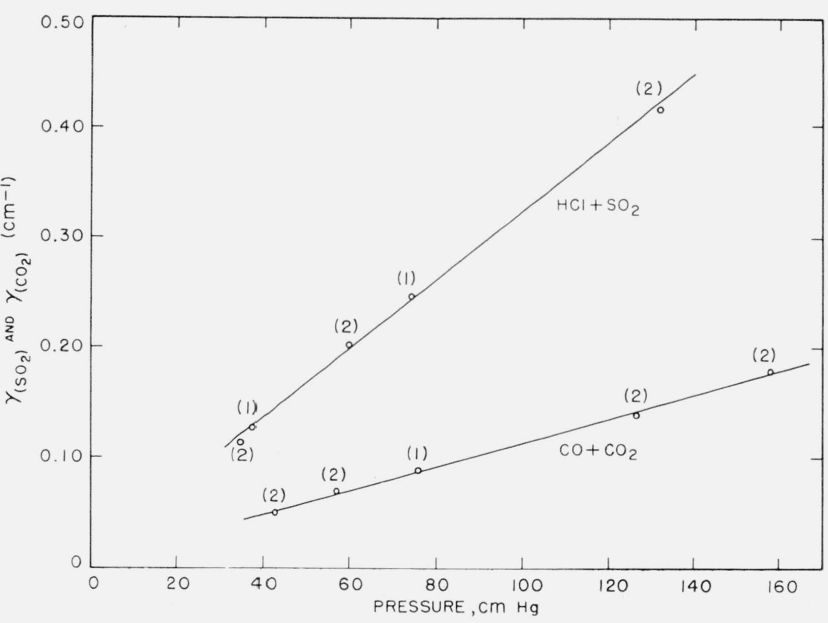

FIgURE 4. The relationship between half-widths and pressure. The $\mathrm{HCl}+\mathrm{SO}_{2}$ line is given for $|m|=2$, and the $\mathrm{CO}+\mathrm{CO}_{2}$ line is given for $|m|=4$. The numbers in parentheses indicate whether the data were obtained from the $2-0$ or the $1-0$ band.

\section{References}

[1] W. S. Benedict, R. Herman, G. Moore, and S. Silverman, Can. J. Phys. 34, 850 (1956).

[2] S. Kimel (Doctorate), Weizmann Inst. of Sci., Rehovoth Israel (1960)

[3] W. S. Benedict, R. Herman, G. Moore, and S. Silverman, Astrophys. J. 135, 1, 277 (1962).

[4] D. H. Rank, W. B. Birtley, D. P. Eastman, B. S. Rao, and T. A. Wiggins, J. Opt. Soc. Am. 50, 1275 (1960).

[5] E. K. Plyler and E. D. Tidwell, Z. Electrochem. 64, 717 (1960).

[6] E. K. Plyler, A. Danti, L. R. Blaine, and E. D. Tidwell, J. Research NBS 64A, (Phys. and Chem.) No. 1, 29 (1960).

[7] E. K. Plyler and L. R. Blaine, J. Research NBS 62, 7 (1959)

[8] J. R. Izatt, Progress Rep., J. Hopkins Univ., Lab. of Astrophys. and Phys. Meteorology (1960).

[9] H. Babrov, G. Ameer, and W. Benesch, J. Mol. Spec. 3, 185 (1959).

[10] P. W. Anderson, Phys. Rev. \%6, 647 (1949).

[11] W. V. Smith and R. Howard, Phys. Rev. 79, 132 (1950).

[12] M. A. Hirshfeld, J. H. Jaffe, and S. Kimel J. Chem. Phys. 32, 297 (1960) 